\title{
Theoretical simulations of oxygen $K$-edge resonant inelastic x-ray scattering of kaolinite
}

\author{
Emelie Ertan, ${ }^{1}$ Victor Kimberg, ${ }^{2,3}$ Faris Gel'mukhanov, ${ }^{2,3}$ Franz Hennies, ${ }^{7}$ Jan-Erik Rubensson, ${ }^{6}$ Thorsten Schmitt, ${ }^{8}$ \\ Vladimir N. Strocov, ${ }^{8}$ Kejin Zhou, ${ }^{8,9}$ Marcella Iannuzzi, ${ }^{5}$ Alexander Föhlisch, ${ }^{4}$ Michael Odelius,,${ }^{1, *}$ and Annette Pietzsch ${ }^{4, \dagger}$ \\ ${ }^{1}$ Department of Physics, Stockholm University, AlbaNova University Center, 10691 Stockholm, Sweden \\ ${ }^{2}$ Theoretical Chemistry and Biology, Royal Institute of Technology, 10691 Stockholm, Sweden \\ ${ }^{3}$ Institute of Nanotechnology, Spectroscopy and Quantum Chemistry, Siberian Federal University, 660041 Krasnoyarsk, Russia \\ ${ }^{4}$ Helmholtz-Zentrum Berlin für Materialien und Energie GmbH, Albert-Einstein-Strasse 15, 12489 Berlin, Germany \\ ${ }^{5}$ Physical Chemistry Institute, University of Zürich, 8057 Zürich, Switzerland \\ ${ }^{6}$ Department of Physics and Astronomy, Uppsala University, 75120 Uppsala, Sweden \\ ${ }^{7}$ MAX IV Laboratory, Lund University, 22100 Lund, Sweden \\ ${ }^{8}$ Paul Scherrer Institut, Swiss Light Source, 5232 Villigen PSI, Switzerland \\ ${ }^{9}$ Diamond Light Source, Ltd., Diamond House, Harwell Science \& Innovation Campus, Didcot, Oxfordshire OX11 ODE, United Kingdom
}

(Received 2 February 2017; revised manuscript received 15 March 2017; published 5 April 2017)

\begin{abstract}
Near-edge x-ray absorption fine structure (NEXAFS) and resonant inelastic x-ray scattering (RIXS) measurements at the oxygen $K$ edge were combined with theoretical spectrum simulations, based on periodic density functional theory and nuclear quantum dynamics, to investigate the electronic structure and chemical bonding in kaolinite $\mathrm{Al}_{2} \mathrm{Si}_{2} \mathrm{O}_{5}(\mathrm{OH})_{4}$. We simulated NEXAFS spectra of all crystallographically inequivalent oxygen atoms in the crystal and RIXS spectra of the hydroxyl groups. Detailed insight into the ground-state potential energy surface of the electronic states involved in the RIXS process were accessed by analyzing the vibrational excitations, induced by the core excitation, in quasielastic scattering back to the electronic ground state. In particular, we find that the NEXAFS pre-edge is dominated by features related to $\mathrm{OH}$ groups within the silica and alumina sheets, and that the vibrational progression in RIXS can be used to selectively probe vibrational modes of this subclass of $\mathrm{OH}$ groups. The signal is dominated by the $\mathrm{OH}$ stretching mode, but also other lower vibrational degrees of freedom, mainly hindered rotational modes, contribute to the RIXS signal.
\end{abstract}

DOI: 10.1103/PhysRevB.95.144301

\section{INTRODUCTION}

High-resolution RIXS has had a large impact on science in the recent years. Information has been gained on potential energy surfaces [1,2], quantum beats [3], parity [4], molecular dynamics [5,6], and the $\mathrm{N}_{2}$ ionization threshold [7]. The investigated systems vary from simple homonuclear molecules, such as $\mathrm{N}_{2}$ [7] and $\mathrm{O}_{2}$ [2,3], to molecular liquids, such as acetone [5] and acetone chloroform [1].

Processes in small molecules can be theoretically fully described; however, for experiments on more complex systems, the question arises of how theory can help the understanding of experiments and give insight into the potential energy surface and nuclear dynamics of these systems. By studying the quasielastic RIXS process, where inhomogeneous electronic broadening is quenched and vibrational resolution can be achieved, we can probe the ground-state potential energy surface, as shown in recent studies on gas phase $[1,2,8]$, on liquids $[1,5,6,9]$, and on solids [1]. By studying the detuned spectra we can get insight into the influence of ultrafast nuclear dynamics in RIXS, something that is especially pronounced in hydrogen-containing systems [10-14].

In this work, we investigate how a $\mathrm{OH}$ group experiences its environment in a solid clay material, kaolinite $\mathrm{Al}_{2} \mathrm{Si}_{2} \mathrm{O}_{5}(\mathrm{OH})_{4}$, which is a layerwise structure made up of silica sheets of tetrahedral coordination $\left(\mathrm{SiO}_{4}\right)$ and alumina sheets of octahedral coordination $\left(\mathrm{AlO}_{6}\right)$. The silica and alumina sheets are connected to each other covalently in layers, and the layers,

\footnotetext{
*Corresponding author: odelius@fysik.su.se

†Corresponding author: annette.pietzsch@helmholtz-berlin.de
}

in turn, interact with hydrogen bonding from hydroxyl groups in the alumina sheet to oxygen atoms situated on the silica sheet in the next layer (-Al-O-H . . O O-Si-), as depicted in Fig. 1 [15]. We can distinguish four inequivalent hydroxyl groups, three in-between the layers and one inside the layer. We compare experimental high-resolution RIXS with high-level spectrum simulations to understand how a selected $\mathrm{OH}$ group is affected by a confined environment.

\section{COMPUTATIONAL DETAILS}

Periodic electronic structure calculations within density functional theory (DFT) were used to derive NEXAFS spectra and in the calculation of both potential energy surfaces used in the simulation of the RIXS spectra. The NEXAFS spectra can be calculated for the static ground-state geometry, but for the vibrationally resolved quasielastic RIXS spectra, we need to simulate the nuclear dynamic response, which requires access to the potential energy surfaces of the electronic ground state and the probed core-excited state. The large number of nuclear degrees of freedom for the crystal supercell makes it impossible to explore the full potentials. Instead, it is necessary to reduce the dimensionality of the problem by investigating only degrees of freedom, which are likely excited in the RIXS process. For this purpose, we will employ various schemes of normal-mode analysis to derive one-dimensional cuts (corresponding to directions in the configurational space along which the system might be excited) through the potential energy surfaces.

For the actual simulation of the RIXS spectra, onedimensional quantum dynamics was used, employing wavepacket propagation to model the time evolution of nuclear degrees of freedom in decaying core-excited intermediate 


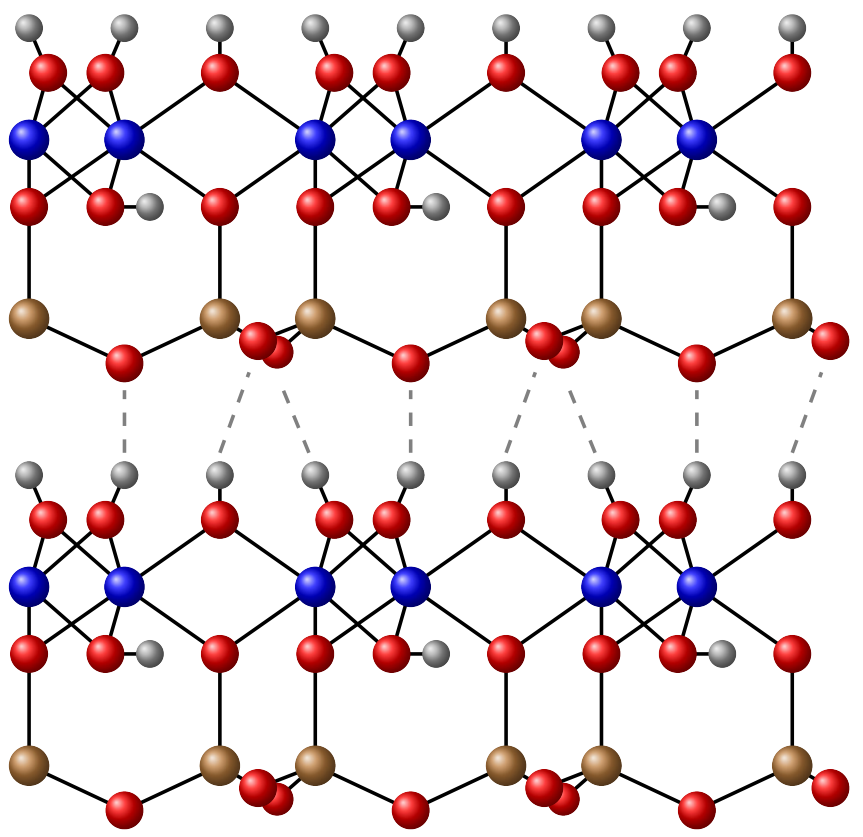

FIG. 1. Layerwise structure of the kaolinite crystal. The elements are color coded: Si (beige), Al (blue), O (red), and $\mathrm{H}$ (gray), and covalent (solid lines) and hydrogen-bonds (dashed lines) are indicated.

states. This procedure is motivated by the local nuclear response to the core excitations.

\section{A. Density functional calculations}

Based on X-ray diffraction data of kaolinite [16], we set up a model consisting of $3 \times 2 \times 2$ copies of the primitive unit cell to form the periodic supercell, resulting in a total system of 408 atoms. For this primitive triclinic supercell, the following unit-cell parameters were used: $a=15.462 \AA$, $b=17.884 \AA$, and $c=14.802 \AA, \alpha=91.69^{\circ}, \beta=104.61^{\circ}$, and $\gamma=89.82^{\circ}$. The structure was optimized, and using the optimized coordinates we calculated the full Hessian (second derivatives of the energy with respect to the nuclear coordinates) numerically through finite differences with a step size of 0.01 a.u. Subsequently, the Hessian was used in combination with different choices of atomic masses to obtain harmonic normal vibrational modes with displacement coordinates suitable to calculate one-dimensional cuts through the ground-state potential $\left(U_{0}\right)$ and the core-excited-state potential $\left(U_{c}\right)$ for modeling the RIXS spectra. This procedure is described below.

In the modeling of the quasielastic RIXS process, we need to follow dynamics on $U_{0}$ and $U_{c}$. For this we used several different schemes, resulting in cuts along different coordinates in the potential energy surfaces:

(i) In Scheme I, we determined the normal modes in the full phonon spectrum by performing an unbiased vibrational analysis on the system, with all atoms having masses according to the weight of the most abundant natural isotope $\left(m_{\mathrm{Si}}=27.977 \mathrm{u}, m_{\mathrm{Al}}=26.982 \mathrm{u}, m_{\mathrm{O}}=15.995 \mathrm{u}\right.$, and $m_{\mathrm{H}}=$ $1.008 \mathrm{u}$ ). These calculations were used for reference.

(ii) In Scheme II, the stretching vibrations of a particular $\mathrm{OH}$ group were decoupled from the vibrations of equivalent
$\mathrm{OH}$ groups in the environment by isotope substitution with deuterium in the other hydroxyl groups. By deuterating the remaining $\mathrm{OH}$ groups, we sought to project out the local contribution in the set of symmetry combinations of $\mathrm{OH}$ vibrations onto the nondeuterated $\mathrm{OH}$ group. Deuterating the hydroxyl groups decouples the $\mathrm{OH}$ stretching mode from the vibrations in the environment, thereby allowing us to study this vibrational mode separately.

(iii) In Scheme III, we aimed to simulate also other degrees of freedom around the core-excited $\mathrm{OH}$ oxygen. The rotational and translational vibrational modes of the $\mathrm{OH}$ group have lower frequency than the stretching mode and couple more strongly to the degrees of freedom of the surroundings. Hence, in Scheme III, the masses of all atoms (except the core-excited $\mathrm{OH}$ group) were increased by a factor of 1000 to dynamically decouple these $\mathrm{OH}$ vibrations and librations from the environment. The large increase in mass effectively freezes the environment and allows us to study the pure rotational and translational vibrations of the $\mathrm{OH}$ group, in addition to the stretching vibration.

The normal vibrational mode calculations of Schemes I-III were performed on the optimized coordinates. The representations in Schemes II and III are justified by the local response to the core excitation in RIXS, and the normal vibrational modes determined were used to derive the potential energy cuts.

One-dimensional cuts in the potential energy surface for the ground state and the core-excited state were calculated along the displacement coordinate, for the most significant vibrational mode for each inequivalent hydroxyl group, obtained from Scheme II in case of the $\mathrm{OH}$ stretching mode and Scheme III for the lower energy vibration modes. Displacement vectors for the vibrational modes in Schemes I-III were expressed in Cartesian coordinates, which, however, creates an artificial coupling between the rotations and the stretching mode for large distortions.

Geometry optimization, vibrational analysis, and potential energy surfaces were calculated in the CPMD [17] code using DFT with a pseudopotential description and a plane-wave basis. The kinetic energy cutoff controlling the plane-wave orbital expansion was set to $85 \mathrm{Ry}$, and semilocal pseudopotentials were used to describe the core electrons. The BeckeLee-Yang-Parr (BLYP) [18,19] functional was employed together with the norm-conserving Troullier-Martins [20] pseudopotential for $\mathrm{O}$ and $\mathrm{H}$ and dual-space Goedecker [21,22] pseudopotentials for $\mathrm{Si}$ and $\mathrm{Al}$. The core-excited states were modelled using a pseudopotential corresponding to the core-ionized oxygen, as previously developed [10], with the addition of the core-excited electron.

For simulation of NEXAFS spectra of the nine crystallographically inequivalent oxygen atoms, we used the half-core-hole $(\mathrm{HCH})$ transition potential method with a mixed pseudopotential and all-electron periodic DFT, as implemented in the Gaussian augmented plane-wave method in the CP2K software [18,19,23-29]. In these periodic DFT calculations, the BLYP functional was employed, together with the local Gaussian 6-311 $++G(2 d, 2 p)$ basis set for the all-electron atoms in the core-excited hydroxyl group or in the core-excited oxygen atom, and double-zeta valence polarized Goedecker-Teter-Hutter (DZVP-GTH) basis sets [30] for the remaining $\mathrm{Al}, \mathrm{Si}, \mathrm{O}$, and $\mathrm{H}$ atoms, which were represented 
by GTH pseudopotentials [21,22]. The kinetic energy cutoff for the electron density was set to 300 Ry. The NEXAFS simulations were performed using coordinates obtained from the geometry optimization described above. The resulting discrete NEXAFS spectra were convoluted with a Gaussian function, with a FWHM of $0.6 \mathrm{eV}$, and for a direct comparison with the experimental NEXAFS spectrum a total calculated spectrum, averaged over the contribution of all individual oxygen atoms, was formed. Also, a constant energy shift of $-3.0 \mathrm{eV}$ was applied to the theoretical NEXAFS spectra to align the computed spectra to the oxygen $K$ edge pre-edge in the experimental spectrum.

\section{B. Quantum nuclear dynamics simulations of RIXS}

Using one-dimensional cuts through the potential energy surfaces derived with the DFT methodology as described in the previous section, the RIXS spectra was simulated employing one-dimensional wave-packet propagation, a method developed for molecular systems [31]. These calculations will now be presented.

The RIXS process in principle involves three electronic states: the initial ground state, the core-excited state which for $\mathrm{O} K$-edge RIXS has a hole in the O $1 s$ core level on a particular oxygen atom, and a final (ground or valence-excited) state, reached through fluorescence decay of the core-excited state.

The RIXS cross section is, in general, given by the well-known Kramers-Heisenberg [32] equation. For analysis of vibrationally resolved RIXS data involving shallow and dissociative potentials, time-dependent quantum dynamics simulations are often more efficient than simulations in the stationary picture, expressed in terms of excitation frequencies, in particular, for analysis of vibrationally resolved RIXS data, as in this study. The Kramers-Heisenberg equation expressed in the time-dependent formalism [33] becomes

$$
\tilde{\sigma}\left(\omega, \omega^{\prime}\right)=\frac{1}{\pi} \operatorname{Re} \int_{0}^{\infty} d \tau \sigma(\omega, \tau) e^{i\left[\omega-\omega^{\prime}-\left(E_{f}^{(0)}-E_{0}^{(0)}\right)\right] \tau},
$$

a half-Fourier transform of the autocorrelation function

$$
\sigma(\omega, \tau)=\langle\Psi(0) \mid \Psi(\tau)\rangle .
$$

$|\Psi(\tau)\rangle=e^{-i\left(\mathbf{H}_{f}-E_{0}^{(0)}\right) \tau} e^{-\Gamma_{f} \tau}|\Psi(0)\rangle$ and the wave packet in the core-excited state, integrated over its full time-evolution on the core-excited-state potential $U_{c}$ is

$$
|\Psi(0)\rangle=\mathbf{Q} \int_{0}^{\infty} d t e^{-i\left(\mathbf{H}_{c}-E_{0}^{(0)}-\omega\right) t} \mathbf{V}|0\rangle e^{-\Gamma_{c} t} .
$$

$\omega$ and $\omega^{\prime}$ are the incoming and scattered x-ray photons. $E_{0}^{(0)}$ and $E_{f}^{(0)}$ are the zero-point energies of the electronic ground state and final state. (In our case of the quasielastic scattering $E_{f}^{(0)} \equiv E_{0}^{(0)}$.)

Here $\mathbf{V}$ and $\mathbf{Q}$ are the excitation and decay operators which contain the dipole transition moments, and $\mathbf{H}_{c}$ and $\mathbf{H}_{f}$ are the Hamiltonian operators of the core-excited and final state, respectively. In the reduced dimension, the stationary initial state (wave packet) $|0\rangle$ is obtained by solution of the timeindependent nuclear Schrödinger equation in the ground-state potential $U_{0}$.

The RIXS intensities are in general dependent on the polarization of the incoming X-ray pulse and the direction of the outgoing fluorescent light. However, for quasielastic scattering, where the core-excited states are isolated in energy, the anisotropy dependence of the RIXS intensities can be neglected. However, near crossings of the excited-state potential energy surfaces, which cause the breakdown of the Born-Oppenheimer approximation, the effect of anisotropy becomes great and needs to be taken into account in Eq. (3).

The nuclear dynamics was simulated using the RAM wave-packet program [31] in which the full RIXS process is simulated. The computation space was defined with $x_{\min }=$ -1.5 a.u., $x_{\max }=9.0$ a.u., with $N=2000$ points and binding energy range of $0-15 \mathrm{eV}$. The final-state lifetime broadening is $\Gamma_{f} \approx 0 \mathrm{eV}$, since the final state is the electronic ground state. In our calculations, however, we need nonzero lifetime broadening, $\Gamma_{f}=10^{-4} \mathrm{eV}$ at half-width at half maximum (HWHM), in order to optimize computational costs. Let us note that this broadening value is much smaller than the broadening resulting from experimental inaccuracy (FWHM = $0.052 \mathrm{eV})$. The core-hole lifetimes for light elements, like oxygen, are predominantly determined by the Auger process [34], leading to electron emission, but the observable in this study is governed by photons, from the minority fluorescence process. The lifetime broadening of the $\mathrm{O} 1 \mathrm{~s}$ core hole is fairly independent of the specific core-excited state and the environment in different molecules; the lifetime broadening of the core-hole in $\mathrm{CO}, \mathrm{H}_{2} \mathrm{O}$, and $\mathrm{O}_{2}$ is $\Gamma_{c}=$ 0.07-0.08 eV (HWHM) [35,36]. We have assumed that the lifetime broadening of the $\mathrm{O} 1 s$ core-hole in kaolinite will have a similar value, $\Gamma_{c}=0.08 \mathrm{eV}$ (HWHM). For the RIXS simulations, the transition dipole moment is assumed to be constant and the temperature-dependent Boltzmann distribution for the initial state is assumed to be negligible for the vibrational modes, i.e., the wave-packet simulation is initialized from a ground-state distribution at $0 \mathrm{~K}$.

\section{EXPERIMENTAL DETAILS}

The commercially available natural kaolinite ASP G90 (BASF Corp.) was kindly donated for the study. We used the highly pulverized kaolinite ASP G90 from BASF, which was compacted before insertion into the sample chamber. The sample was kept in a dry atmosphere to prevent contamination with water. The sample was further outgassed during pumpdown and the NEXAFS and RIXS measurements were performed in vacuum with pressures $<5 \times 10^{-8}$ mbar.

The kaolinite measurements were performed at the SAXES spectrometer [37] at the ADRESS beamline [38] at the Swiss Light Source. The overall energy resolution was less than $50 \mathrm{meV}$. The angle of incoming and outgoing radiation was $45^{\circ}$ each with the total scattering angle being $90^{\circ}$. The energy calibration of the spectra was done in two steps: the absolute energy was defined by the $\mathrm{CO}$ absorption energy, while the energy dispersion in the RIXS spectra was derived with the help of RIXS on $\mathrm{O}_{2}$ gas using its well-known vibrational progression [2,39]. The on-resonance RIXS spectrum has previously been published by Schreck et al. [1].

\section{RESULTS AND DISCUSSION}

In kaolinite [16], there are in total four types of crystallographically inequivalent hydroxyl groups in the primitive unit cell, one [denoted $\mathrm{OH}(1)$ ] situated in the intermediate region 


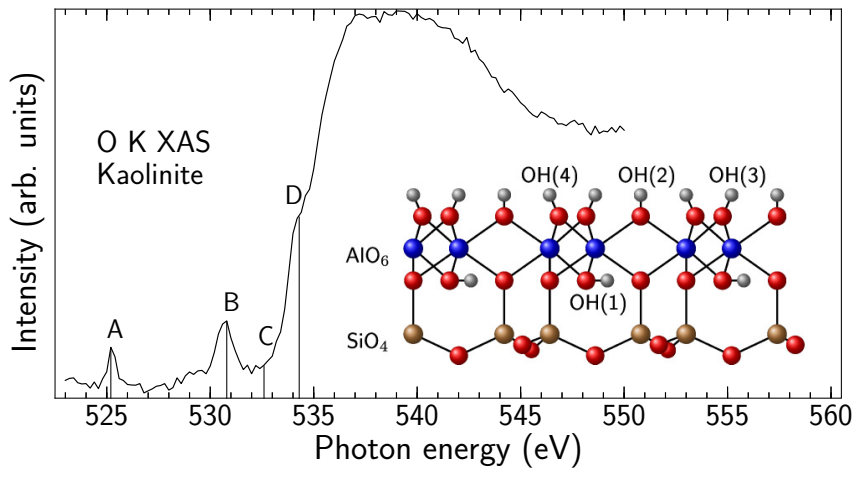

FIG. 2. Experimental oxygen $K$-edge NEXAFS spectrum of kaolinite. Particular pre-edge features are marked to facilitate the presentation. Inset: $\mathrm{OH}$ groups in kaolinite and their confinement.

of the covalently connected silica and alumina sheets and the other three [denoted $\mathrm{OH}(2-4)$ ] serving as hydrogen bond connectors between the layers (see inset in Fig. 2). The lattice parameters and initial crystal coordinates that we use are those determined with x-ray diffraction by Neder et al. [16], which imply that the hydrogen positions are associated with higher uncertainty. The unit-cell coordinates from the experiment [16] and those derived from the optimization in the $3 \times 2 \times 2$ supercell in this work are displayed in Table I in Appendix B. We adopt the notation of the hydroxyl groups from Neder et al. [16].

\section{A. O $K$-edge NEXAFS}

We performed NEXAFS and RIXS measurements at the $\mathrm{O} K$-edge of the kaolinite to have a site-selective and atomspecific measurement of the $\mathrm{OH}$ groups. The experimental $\mathrm{O} \mathrm{K}$ NEXAFS is shown in Fig. 2. We observe, apart from the main resonance, three distinct features $\mathrm{A}, \mathrm{B}$, and $\mathrm{D}$. Features A and B are assigned to defect states and radiationinduced production of quasimolecular oxygen and have been discussed extensively in Ref. [40]. The shoulder feature D, however, located at $534.3 \mathrm{eV}$, arises from the $\mathrm{OH}$ groups. The adjacent energy, denoted $\mathrm{C}(532.9 \mathrm{eV})$, among other possible contributions, includes a signal from detuning of the core-excited state responsible for the hydroxyl group feature D. To understand and disentangle the contribution of the four different inequivalent $\mathrm{OH}$ groups to these features, we calculated NEXAFS spectra for all crystallographically inequivalent oxygen atoms in kaolinite.

The calculated NEXAFS spectrum is shown in Fig. 3. The respective coordinates are given in Table I in Appendix B. $\mathrm{O}(1-5)$ correspond to the nonhydroxyl oxygen atoms bonded to the $\mathrm{Al}$ and $\mathrm{Si}$ atoms in the crystal. In Fig. 3(a), the separate contributions of the different $\mathrm{OH}$ and $\mathrm{O}$ species are shown and related to the experiment. We find that feature D in the experimental spectrum consists mainly of a transition to core-excited states at the $\mathrm{OH}(1)$ oxygen, which is located inside the clay sheets. In Fig. 3(b), the contribution of this hydroxyl group is compared to the computed average and the experimental NEXAFS spectrum of kaolinite. The reason for the dominance of $\mathrm{OH}(1)$ at the pre-edge is interpreted to be its position in between the sheets in the crystal, which is limiting the ability of this hydroxyl group to form hydrogen bonds
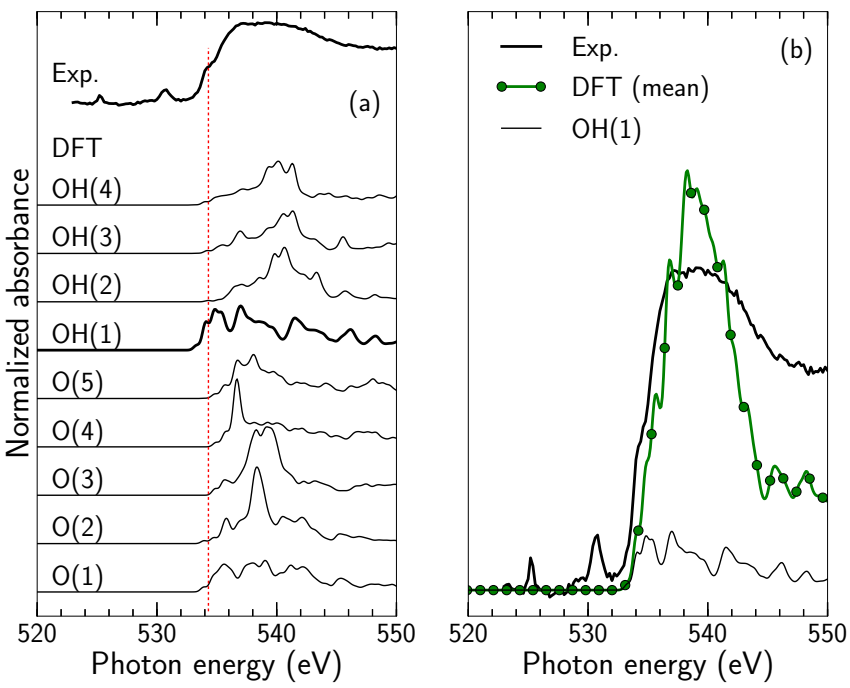

FIG. 3. (a) Experimental NEXAFS and the computed (DFT$\mathrm{HCH}$ ) NEXAFS spectra displaying the contribution of the chemically inequivalent oxygen atoms to the total absorption spectrum. The excitation energy of the pre-edge feature $\mathrm{D}$ is marked by a dashed red line. (b) The experimental NEXAFS spectrum compared to the average of individual computed spectra and the contribution of hydroxyl oxygen in $\mathrm{OH}(1)$, see inset in Fig. 2.

with the environment. The signature of NEXAFS pre-edge features for weakly hydrogen-bonding $\mathrm{OH}$ groups has been established for liquid water [41]. However, the assignment has some uncertainty, since the computed average does not faithfully reproduce the experimental NEXAFS due to the limited accuracy of the DFT HCH transition potential method. Nevertheless, this is a strong indication that by $\mathrm{x}$-ray core excitation at $\mathrm{D}$, we can selectively probe the $\mathrm{OH}(1)$ group and study it in greater detail.

\section{B. O $K$-edge RIXS on-resonance and detuned}

To investigate the chemical environment of the $\mathrm{OH}(1)$ group, we measured RIXS against the absorption feature D. The local nature of the RIXS excitation means that by selectively exciting at a certain resonance we can observe the signal from that specific atom, in this case the oxygen atom from $\mathrm{OH}(1)$. The resulting RIXS spectrum gives not only a picture of the local electronically excited state, but also allows us to map the ground-state potential energy surface using information on the local vibrational excitation created in the RIXS process [5,6]. In our measurements, apart from the electronically excited states at 7-14-eV loss, we observe a clear vibrational progression close to the elastic line at $0-3-\mathrm{eV}$ loss (see Fig. 4). For excitation on the resonance of feature D, we observe four to five overtones, each of them consisting of a double peak with $\sim 0.1-\mathrm{eV}$ splitting [see Fig. 4(b)]. The RIXS spectrum from the lower photon energy in $\mathrm{C}$ shows a shorter vibrational progression, which is discussed below.

The vibrational spectra of the $\mathrm{OH}$ groups from Scheme I is discussed in Appendix A, but for the simulations of RIXS spectra we have used Schemes II-III.

Using Scheme II of the vibrational normal-mode analysis, we obtained the normal-mode vectors of the $\mathrm{OH}(1)$ and one-dimensional cuts in the ground state, and the 

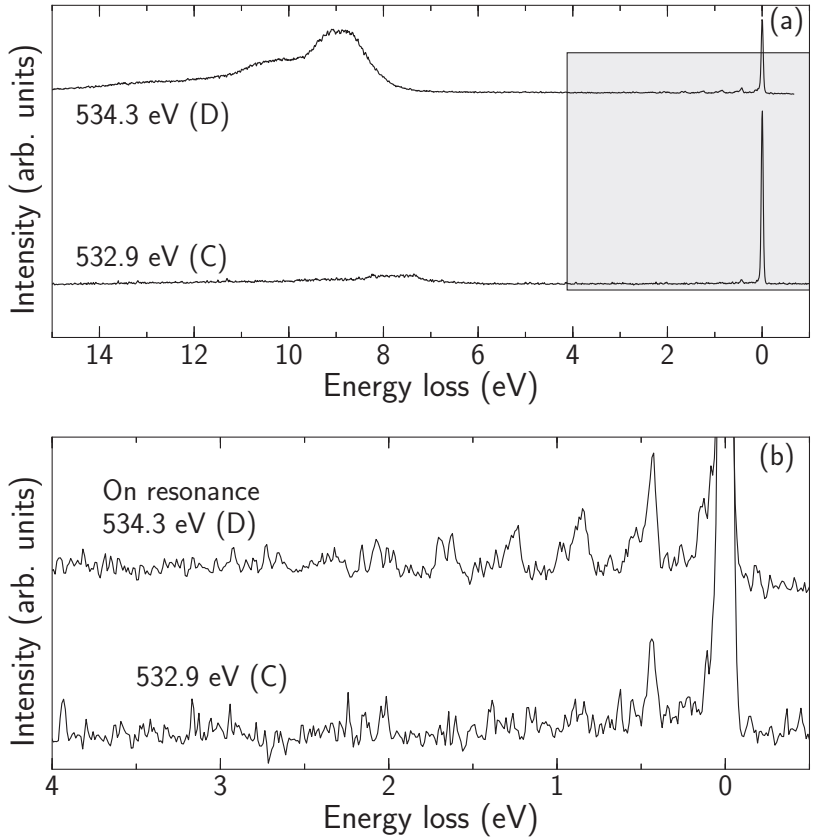

FIG. 4. Experimental RIXS spectra measured for incoming radiation frequencies corresponding to $\mathrm{D}(534.3 \mathrm{eV})$ and $\mathrm{C}(532.9 \mathrm{eV})$. The spectra are normalized against the measurement time (20 min for feature $\mathrm{C}$ and $70 \mathrm{~min}$ for feature D). (a) Full experimental RIXS spectrum at C and D. (b) RIXS spectra zoomed in to display vibrational progression.

core-excited-state potential energy surfaces were calculated along the $\mathrm{OH}$ stretching coordinate [see Fig. 5(a)].

Both ground-state potential and core-excited-state potential cuts are bound along the $\mathrm{OH}$ stretching mode, which at large $\mathrm{OH}$ distance is due to repulsive interactions between the stretched hydroxyl group and the environment. The equilibrium $\mathrm{OH}$ distance in the core-excited-state potential, $\mathrm{rOH}_{\text {core-exceq }}=1.957 \AA$, is strongly elongated in comparison to the electronic ground state, $\mathrm{rOH}_{\mathrm{gs}, \mathrm{eq}}=0.975 \AA$, where the core-excited-state potential has a large gradient, meaning that there will be an ultrafast elongation of the $\mathrm{OH}$ bond during the initial nuclear dynamics. The potential cuts along the stretching modes are only harmonic at small displacement around the minima and become anharmonic for $\mathrm{OH}$ bond elongation, where the stretching mode also couples to other vibrational modes.

The elongated RIXS spectrum, as simulated for the potential energy surfaces in Fig. 5(a), is shown on an energy-loss scale in Figs. 5(b) and 5(c). The RIXS spectrum corresponding to excitation to a vibrationally hot core-excited state is shown, as determined from the quantum nuclear dynamics simulations in the RAM wave-packet program of the x-ray absorption signal from the potential cuts in Fig. 5.

First we will make a small sidetrack to discuss the strong features appearing at $\sim 7 \mathrm{eV}$ in the calculated RIXS spectra in Fig. 5(b). According to our one-dimensional simulations, the core-excited wave packet is able to reach a right classical turning point (large bond length) of the potential within the core-hole lifetime. The integrated wave packet Eq. (3) has a local maximum at the classical turning point [see blue line
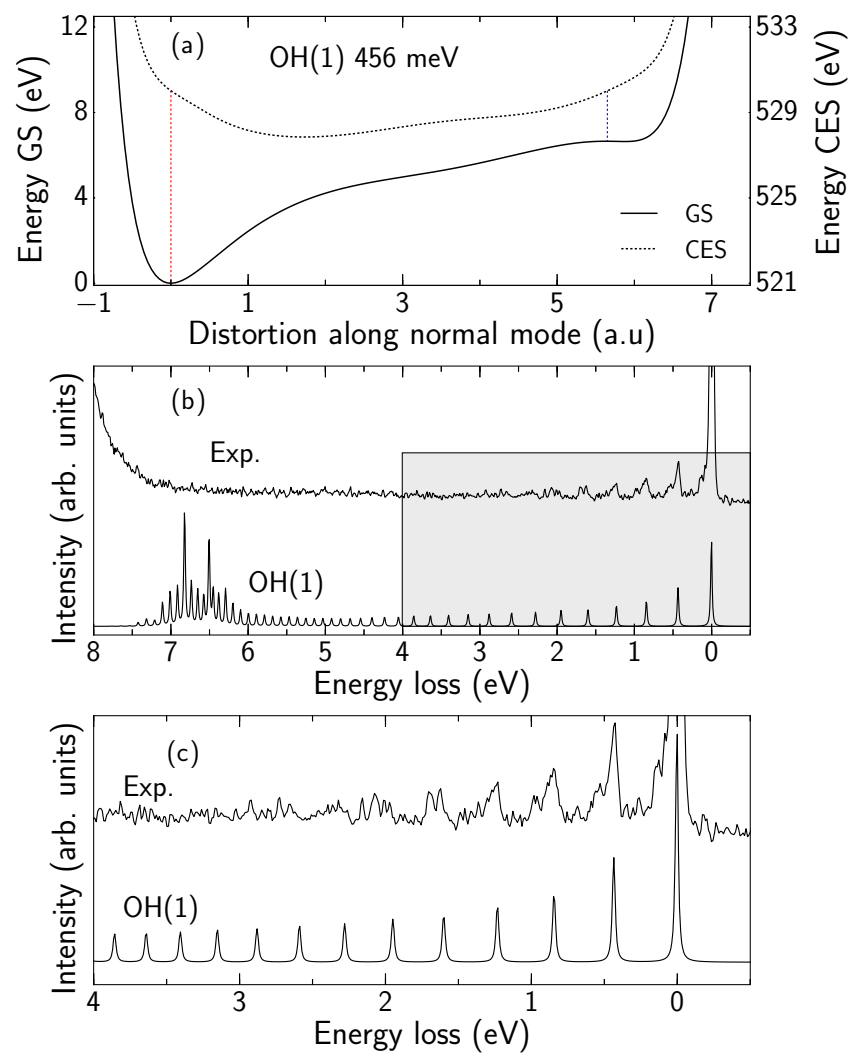

FIG. 5. (a) Cuts along the $\mathrm{OH}$ stretching mode of the potential energy surface of the ground state and the first core-excited state (CES) for $\mathrm{OH}(1)$. Vertical excitation from ground-state equilibrium and classical turning point is shown by vertical red and blue dashed lines. (b) Calculated RIXS spectra at the on-resonance frequency compared to experimental RIXS spectra. The intensity of the calculated RIXS spectra are normalized against the intensity of the experimental $\left(v_{0} \rightarrow 1\right)$ transition. Notice that the emerging increase at 7-8 eV energy loss in the experimental spectrum corresponds to the onset of the first electronically excited state. (c) Calculated and experimental spectra zoomed in on the $-0.5-4-\mathrm{eV}$ energy-loss region.

Fig. 5(a)], resulting in the strong RIXS feature at around $7 \mathrm{eV}$, as follows from the reflection principle [42]. While we see these features in the simulated RIXS spectra, they are quenched in the experimental spectrum. One explanation for the quenching in the experiment would be that the other hydroxyl groups will contribute to smearing out of the turning point intensity in the spectrum, which is discussed further in Appendix A. However, we have shown in this paper that the predominating hydroxyl group at $\mathrm{D}$ is $\mathrm{OH}(1)$, which makes this explanation unlikely. Instead we propose that these RIXS features are effected by low-energy vibrations.

Returning to the RIXS spectra in Figs. 5(b) and 5(c), we see an extended vibrational progression in the simulated RIXS spectrum. The simulated data is in good agreement with the energy loss seen in the main experimental peaks in the $0-4-\mathrm{eV}$ energy-loss region. However, the double peak, observed experimentally, is not present in the calculated RIXS spectra. We propose to ascribe the fine structure in the experimental RIXS peaks to the vibrational excitations in other degrees of freedom. Hence below, we have investigated the 

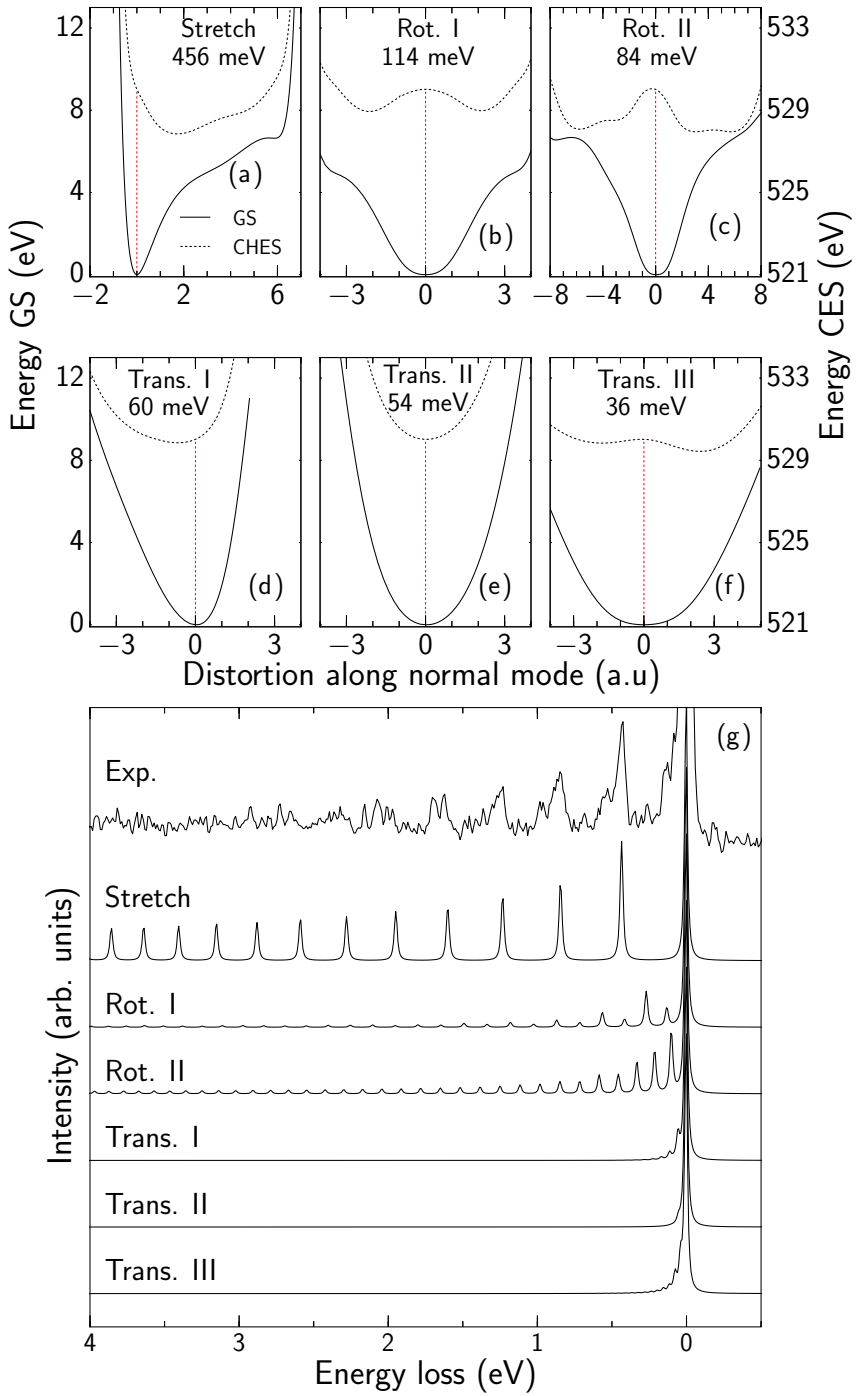

FIG. 6. Two top panels (a-f): Cuts in the potential energy surface of the ground state (solid line) and the first core-excited state (dashed line) along all six vibrational modes of $\mathrm{OH}(1)$ in a frozen environment. Vertical red dashed lines show the vertical transition from the groundstate minimum. (g) Separately calculated on-top resonance RIXS spectra of $\mathrm{OH}(1)$ for cuts along each six vibrational mode compared to experimental RIXS spectrum. Calculated spectra are internally normalized against elastic $\left(v_{0} \rightarrow 0\right)$ transition.

energy channels opened by $\mathrm{OH}$ rotational and $\mathrm{OH}$ translation modes in the simulated RIXS spectra.

To extract the lower frequency vibrations of the $\mathrm{OH}(1)$ hydroxyl group, we performed a normal-mode analysis according to Scheme III. Using this setup, six $\mathrm{OH}$ vibrational modes were resolved for $\mathrm{OH}(1)$ (in frozen environment $3 N$ $=6$, for $N=2$ ). These $\mathrm{OH}$ vibrations are visualized in Fig. 10 in Appendix B, and the vibrational frequencies are reported in and Table III in Appendix B. Cuts in the potential energy surface of the ground state and the core-excited state calculated along these six normal-mode coordinates are displayed in Figs. 6(a)-6(f). The cut along the $\mathrm{OH}$ stretching coordinate in the potential energy surface (a) is essentially identical in Schemes I, II, and III, indicating that the effect of additional freezing of the environment is negligible for this mode. This is supported by the $\mathrm{OH}(1)$ phonon density of states, displayed in Fig. 11 in Appendix B, where we see that the vibrational frequencies of the $\mathrm{OH}(1)$ stretching mode remain essentially unaffected for all three schemes. For the low-frequency modes, the change in the phonon dispersion is due to the projection required to study localized modes.

The cuts in the potential energy surface along the different vibrational modes vary greatly with the degree of freedom. Due to its confinement in the crystal, the hydroxyl group is affected by the environment of the surrounding atoms, which determines the shape of the potential. The repulsive caging effect and attractive electrostatic interactions experienced by the hydroxyl group therefore vary with the degree of freedom. In particular, the change in the dipole moment of the $\mathrm{OH}$ group induced by the core excitation strongly influences the interactions. Together, this results in differences in the energy landscapes of the ground and core-excited state for the different degrees of freedom.

In the case of the translation modes I-III of the $\mathrm{OH}$ group, Figs. 6(d)-6(f), the potential shapes vary with the direction of the translation. However, the combination of a small gradient in the core-excited-state potential for the vertical excitation and a large reduced mass (Table III in Appendix B) suggests limited vibrational excitations. In the corresponding RIXS simulations [Fig. 6(g)], we indeed see that the progression in each translational mode is very small. Thereby it is deemed unlikely that these modes give rise to the double peak in the experimental RIXS spectrum. From the distortion vectors of the translational normal modes we observe that they are not pure translations, which also can be deduced from the reduced masses listed in Table III.

On the other hand, the cuts in the core-excited-state potential along rotational modes I and II are more interesting. They are displayed in Figs. 6(b) and 6(c) and both exhibit a doublewell shape. The dipole moments of the $\mathrm{OH}$ groups are reverted by the core excitation; hence, the ground-state geometry, in which the $\mathrm{OH}$ group is pointing to optimize the electrostatic interaction with the connected cations, becomes unfavorable, which explains the double minimum. However, the precise positions of the minima in the potentials for the rotational modes depend on the coupling to the stretching mode, since the displacement vectors are expressed in Cartesian coordinates.

The rotational modes I and II give overlapping vibrational progressions, as seen in Fig. 6(g), and energy splitting of the vibrational peaks of the rotational is smaller than that of the stretching. This is indicating that the double peak in the experimental spectrum, that could not be accounted for by stretching alone, is indeed due to a combination of the stretch with rotational excitations.

Finally, we investigate the effect of varying the excitation energy for a given resonance. By detuning from the resonance, we can obtain further information about dynamics in the coreexcited state of the $\mathrm{O}$ atom. Large detuning effectively shortens the core-excited state lifetime and a detuned spectrum will thus give us a picture of the system at an earlier time after the core excitation. From there, we can deduce how the system evolves after core excitation. The experimental RIXS spectra, on-resonance and detuned, are displayed in Figs. 4 and 7. $\Omega$ is the detuning from the vertical transition $\Omega=E_{c}\left(R_{0}\right)-$ $E_{0}\left(R_{0}\right)$, where $E_{c}$ and $E_{0}$ are the potential energy curves of 


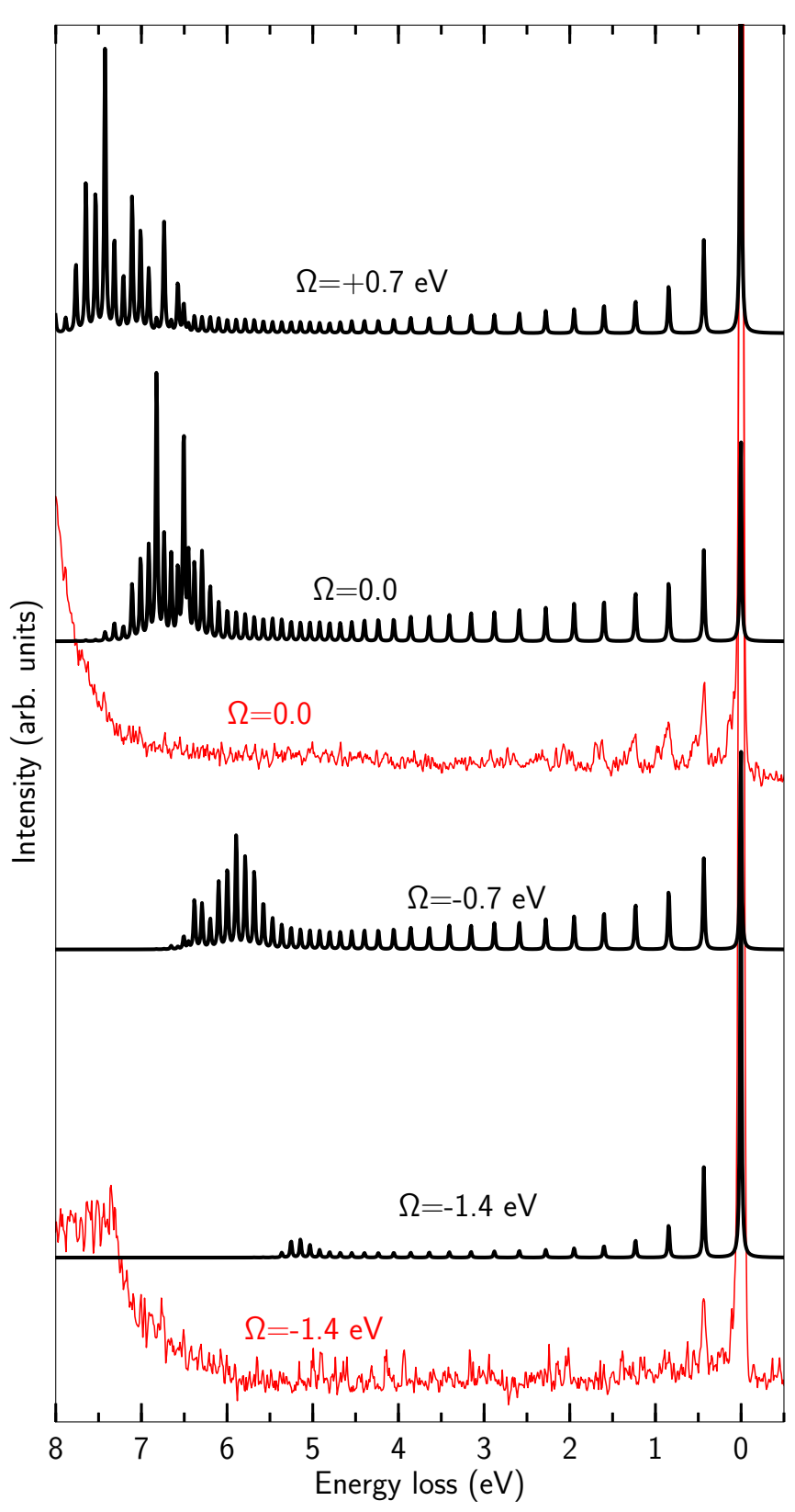

FIG. 7. Calculated RIXS spectra of $\mathrm{OH}(1)$ stretching at incoming photon frequencies on-resonance and detuned from resonance (black) compared to the experimental spectra at $\mathrm{C}$ and $\mathrm{D}$ (red), where the signal at energy losses larger than $7 \mathrm{eV}(\Omega=0.0 \mathrm{eV})$ and $6 \mathrm{eV}(\Omega=-1.4 \mathrm{eV}$ detuned) corresponds to electronic valence excitations. Calculated RIXS spectra are normalized against the intensity of the experimental $\left(v_{0} \rightarrow 1\right)$ transition.

the core-excited and ground states, and $R_{0}$ is the ground-state equilibrium geometry. In the case of $\Omega=-1.4 \mathrm{eV}$ we see that the intensity of the higher vibrational overtones is quenched but also that the shorter propagation time leads to suppression of the slower rotational excitations; the double peak disappears and only a single peak remains.

In Fig. 7, the simulated spectra at $\mathrm{OH}(1)$ for different detunings are displayed. We see that the turning point features in the simulated RIXS spectra move from $\sim 7-\mathrm{eV}$ energy loss to $\sim 6-\mathrm{eV}$ energy loss for $\Omega=-0.7 \mathrm{eV}$ and to $\sim 5-\mathrm{eV}$ energy loss for $\Omega=-1.4 \mathrm{eV}$, with weakening of the intensity of the vibrational overtones in both spectra. This behavior is consistent with the wave packet excited at lower energy reaching the right classical turning point at a shorter bond length, where the energy is smaller [see Fig. 5(a)]. This, in principle, allows to map the energy distance between the core-excited and ground-state potentials.

\section{CONCLUSION}

To understand how water reacts with clay materials is a major question. A first step is to investigate how the dry clay layers interact and how the oxygen inside contributes to the bonding. Thus, mapping the potential energy surface (PES) around the oxygens will tell us about the possible reaction paths. In the next step, water inside the clay could be studied. Its modification of the PES will give insight into the bonding and dynamics of water capture in clay material.

In this study we have mapped the potential energy landscape of hydroxyl groups in kaolinite using NEXAFS and high-resolution RIXS, combined with the quantum chemistry calculations and nuclear wave-packet propagation. We have seen that only the hydroxyl group situated in between the $\mathrm{AlO}_{6}$ and $\mathrm{SiO}_{4}$ sheets $[\mathrm{OH}(1)]$ contributes to the NEXAFS pre-edge and consequently, it is the vibrational modes of this hydroxyl group subclass that are probed by the vibrational progression in the corresponding RIXS spectrum. While the $\mathrm{OH}(1)$ stretching mode is the principal excitation, is also clear that $\mathrm{OH}(1)$ rotational modes influence the RIXS spectra of kaolinite. Exploration of the ground-state and core-excited state potential energy surface along the different vibrational modes of $\mathrm{OH}(1)$ revealed that the energy landscape is very complex. The dynamics of an $\mathrm{OH}$ group is strongly dependent on its environment and even local distortions are affected due to caging effects and changes in the dipole moment of the $\mathrm{OH}$ group at core excitation.

In the theoretical spectra of the $\mathrm{OH}$ stretching modes, there are strong features at high energy loss resulting from the decay of the wave packet near the classical turning point on the core-excited potential energy surface; nevertheless, we see no corresponding features in the experimental spectrum. The anharmonic behavior of the potential energy surface along the stretching and rotational coordinates, which may not be sufficiently represented at large separation in the Cartesian normal modes in Schemes I, II, and III, could explain why we do not see a quenching of the turning point in the simulations. We suggest that a multimode study of the stretching mode and the two rotational modes is needed in the endeavor to faithfully reproduce the experimental spectra. A future prospect is therefore to investigate the coupling effects of the different vibrational modes by a multidimensional study including anharmonic effects. This would potentially give both further insight into the absence of the turning point feature and a more accurate description of the fine structure in the vibrational progression in the experimental RIXS data.

\section{ACKNOWLEDGMENTS}

The project has financial support from the Swedish Research Council (V.R.) and the Carl Trygger Foundation. M.O. 
and A.F. acknowledge partial funding by the Helmholtz Virtual Institute VI419, "Dynamic Pathways in Multidimensional Landscapes." A.F. acknowledges funding from the ERCADG-2014 Advanced Investigator 240, Grant No. 669531, EDAX under the Horizon 2020 EU Framework, Programme for Research and Innovation. V.K. acknowledges support from the Knut and Alice Wallenberg Foundation (Grant No. KAW-2013.0020). V.K. and F.G. acknowledge support within the State contract of the Ministry of Education and Science of the Russian Federation for Siberian Federal University for Scientific Research in 2017-2019 (Project No. 3.2662.2017). The experiments were performed at the ADRESS beam line of the Swiss Light Source at the Paul Scherrer Institut. The computations were partly performed on resources provided by the Swedish National Infrastructure for Computing (SNIC) at the Swedish National Supercomputer Centre (NSC), the High Performance Computer Centre North (HPC2N), and Chalmers Centre for Computational Science and Engineering (C3SE).

\section{APPENDIX A: RESULT OF RIXS ON OH(2-4)}

The vibrational analysis using Scheme I showed an energy dispersion of the stretching vibrations of the different hydroxyl groups of the same type, with maximal dispersion of $0.3 \mathrm{meV}$ for modes with a strong contribution from $\mathrm{OH}(1), 3.4 \mathrm{meV}$ for $\mathrm{OH}(2), 0.6 \mathrm{meV}$ for $\mathrm{OH}(3)$, and $0.9 \mathrm{meV}$ for $\mathrm{OH}(4)$ (see Fig. 8). The small dispersion, especially in the case of $\mathrm{OH}(1)$ and $\mathrm{OH}(3)$, implies that the symmetry combinations of these $\mathrm{OH}$ stretching vibrations in the supercell, corresponding to the phonon dispersion of those modes, are more or less degenerate, and the coupling between the different hydroxyl groups is small.

The harmonic vibrational frequencies for the stretching mode of the different hydroxyl groups, calculated using

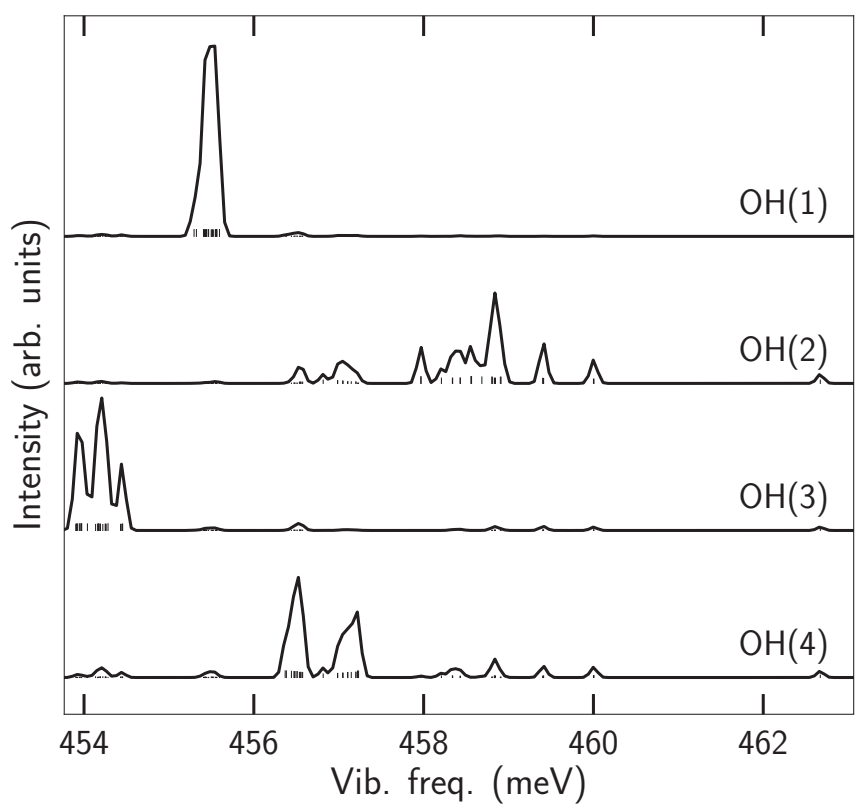

FIG. 8. Energy dispersion of the $\mathrm{OH}(\#)$ contribution as measured by the magnitude of the hydrogen component of the displacement vector for the different stretching modes. The discrete spectra were convoluted with a Gaussian function (FWHM of $0.8 \mathrm{eV}$ ).
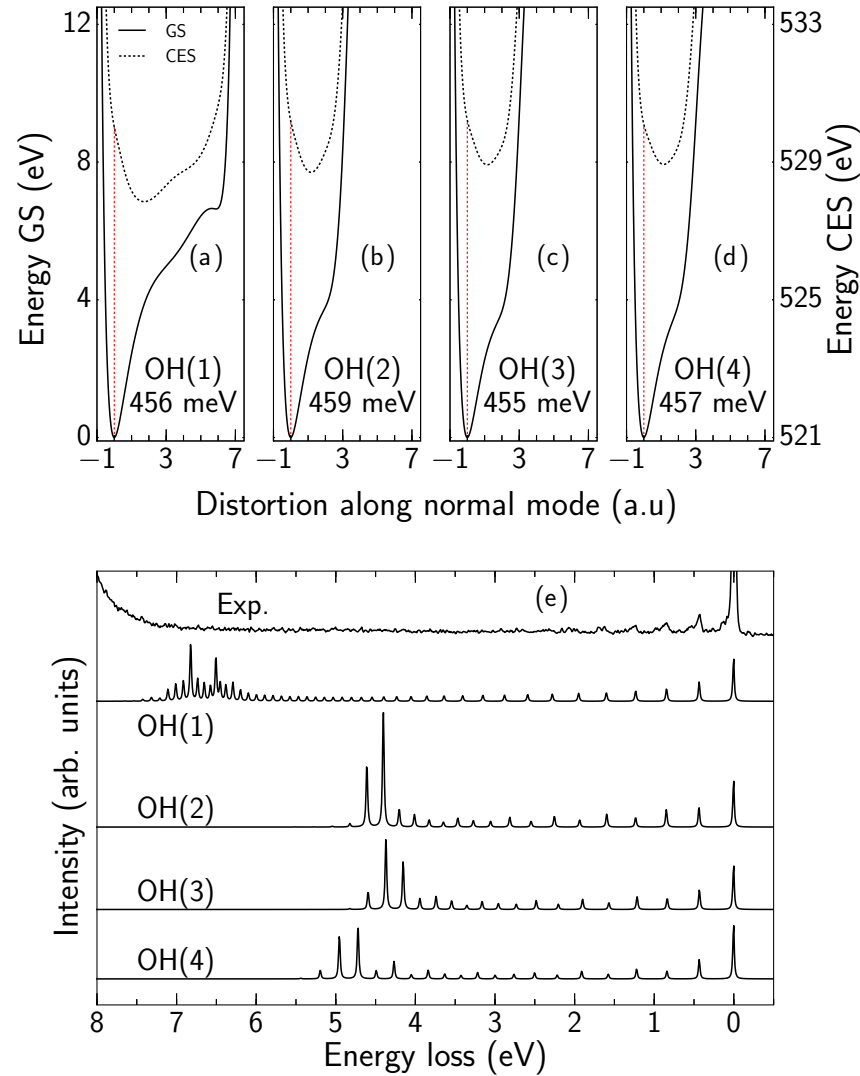

FIG. 9. (a) Cuts along the $\mathrm{OH}$ stretching mode of the potential energy surface of the ground state and the first core-excited state (CES) for $\mathrm{OH}(1-4)$. Vertical excitation from ground-state equilibrium and classical turning point is shown by vertical red dashed lines. (b) Calculated RIXS spectra at the on-resonance frequency compared to experimental RIXS spectra. The intensity of the calculated RIXS spectra are normalized against the intensity of the experimental $\left(v_{0} \rightarrow 1\right)$ transition. (c) Calculated and experimental spectra in the $-0.5-4.0-\mathrm{eV}$ energy-loss region.

Scheme II, exhibit variations within $\pm 3 \mathrm{meV}$, depending on the environment in the crystal (see Table II in Appendix B). Given the FWHM of $67 \mathrm{meV}$ (which is slightly larger than the overall energy resolution of $52 \mathrm{meV}$ ) of the vibrational features in the measured RIXS data, we deemed that the variations and the possible error in calculated stretching frequency were acceptable.

According to the analysis of the computed NEXAFS spectra above, only $\mathrm{OH}(1)$ contributes to the pre-edge. Nevertheless, since oxygen $K$-edge RIXS may probe an extended region of ground-state potential energy surface [1], it is interesting to also look at $\mathrm{OH}(2-4)$ in more detail. As in the case of $\mathrm{OH}(1)$, both ground-state potential and core-excited-state potential cuts are bound along the $\mathrm{OH}(2-4)$ stretching modes, but compared to the ground state, $\mathrm{rOH}_{\mathrm{gs}, \mathrm{eq}}=0.972,0.973$, $0.972 \AA$, the $\mathrm{OH}$ distances of the core-excited-state potentials are elongated, respectively, $\mathrm{rOH}_{\text {core-exc,eq }}=1.617,1.619$, $1.617 \AA$, inducing some ultrafast dynamics in the core-excited state also for $\mathrm{OH}(2-4)$, though not as significant as in the case of $\mathrm{OH}(1)$. 
Energy-loss RIXS spectra simulated for the potential energy surfaces are shown in Fig. 9(e). We see the turning point features in the simulated RIXS spectra also for $\mathrm{OH}(2-4)$ and a progression of vibrational excitations, but exciting fewer overtones than that of $\mathrm{OH}(1)$. The difference in the turning point energy and vibrational progression of $\mathrm{OH}(1)$ and $\mathrm{OH}(2-4)$ is due to the different shape of the potential energy surface cuts. Due to their placement in the crystal, the potential energy surfaces, and hence the simulated RIXS spectra, for $\mathrm{OH}(2-4)$ are naturally very similar to each other.

\section{APPENDIX B: COMPLEMENTARY INFORMATION}

TABLE I. Geometrical parameters for the kaolinite unit cell reproduced from Neder et al. [16] and parameters calculated in this work (both presented in fractional coordinates). The estimated maximal deviation of symmetry equivalent coordinates within the supercell is $\pm 2.2 \times 10^{-2} \AA$.

\begin{tabular}{|c|c|c|c|c|c|c|}
\hline \multirow[b]{2}{*}{ Atom } & \multicolumn{3}{|c|}{ Experimental } & \multicolumn{3}{|c|}{ Calculated } \\
\hline & $X$ & $Y$ & $Z$ & $X$ & $Y$ & $Z$ \\
\hline $\mathrm{Al}(1)$ & 0.2986 & 0.4955 & 0.475 & 0.2904 & 0.4942 & 0.4833 \\
\hline $\operatorname{Al}(1)$ & -0.2063 & 0.3306 & 0.4744 & -0.2175 & 0.3303 & 0.4807 \\
\hline $\operatorname{Si}(2)$ & -0.0032 & 0.3383 & 0.0924 & -0.0116 & 0.3384 & 0.0941 \\
\hline $\operatorname{Si}(2)$ & -0.4892 & 0.1668 & 0.0938 & -0.4982 & 0.1671 & 0.0956 \\
\hline $\mathrm{O}(1)$ & 0.0503 & 0.3538 & 0.3161 & 0.0428 & 0.3534 & 0.3177 \\
\hline $\mathrm{O}(2)$ & 0.1217 & -0.3373 & 0.3166 & 0.1108 & -0.3361 & 0.3188 \\
\hline $\mathrm{O}(3)$ & 0 & 0.5 & 0 & 0 & 0.5 & 0.0 \\
\hline $\mathrm{O}(4)$ & 0.2103 & 0.2318 & 0.0244 & 0.1962 & 0.2277 & 0.0231 \\
\hline $\mathrm{O}(5)$ & 0.2037 & -0.2361 & 0.0003 & 0.1900 & -0.2309 & 0.0006 \\
\hline $\mathrm{OH}(1)$ & 0.0504 & -0.0313 & 0.3253 & 0.0436 & -0.0286 & 0.3318 \\
\hline $\mathrm{OH}(2)$ & -0.0411 & 0.1657 & -0.3957 & -0.0521 & 0.1664 & -0.3904 \\
\hline $\mathrm{OH}(3)$ & 0.0373 & 0.4732 & -0.3959 & 0.0263 & 0.4728 & -0.3905 \\
\hline $\mathrm{OH}(4)$ & 0.0364 & -0.1436 & -0.3920 & 0.0263 & -0.1440 & -0.3843 \\
\hline $\mathrm{H}(1)$ & 0.1423 & 0.0353 & 0.3474 & 0.1385 & 0.0659 & 0.3341 \\
\hline $\mathrm{H}(2)$ & 0.056 & 0.180 & -0.299 & 0.0503 & 0.1713 & -0.2607 \\
\hline $\mathrm{H}(3)$ & 0.036 & 0.486 & -0.292 & 0.0369 & 0.5017 & -0.2611 \\
\hline $\mathrm{H}(4)$ & 0.033 & -0.205 & -0.302 & 0.0374 & -0.1830 & -0.2614 \\
\hline
\end{tabular}

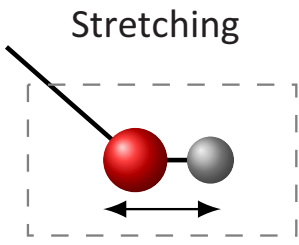

Trans. I
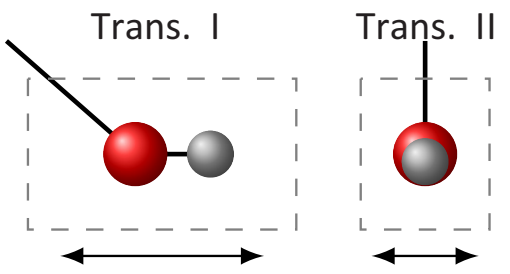

Trans. III

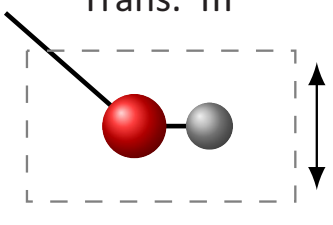

FIG. 10. Visualization of the normal vibrational modes of the $\mathrm{OH}(1)$ group. Top panel shows left-to-right $\mathrm{OH}$ stretching mode, rotation mode I and rotation mode II. Bottom panel shows from left to right translation mode I, translation mode II, and translation mode III.
TABLE II. Vibrational frequencies for the four inequivalent hydroxyl groups calculated in this work using normal-mode analysis according to Scheme II.

\begin{tabular}{llr}
\hline \hline & \multicolumn{2}{c}{ OH stretching frequencies } \\
\cline { 2 - 3 } Group & $\mathrm{cm}^{-1}$ & $\mathrm{meV}$ \\
\hline $\mathrm{OH}(1)$ & 3674 & 456 \\
$\mathrm{OH}(2)$ & 3700 & 459 \\
$\mathrm{OH}(3)$ & 3666 & 455 \\
$\mathrm{OH}(4)$ & 3683 & 457 \\
\hline \hline
\end{tabular}

TABLE III. All vibrational frequencies for the hydroxyl group $\mathrm{OH}(1)$ and reduced mass calculated in this work using normal-mode analysis according to Scheme III (see Fig. 10). The frequency of the stretching mode calculated with this setup is the same as obtained for this hydroxyl group using Scheme II.

\begin{tabular}{lrrc}
\hline \hline & \multirow{2}{*}{$\mu$} & \multicolumn{2}{c}{$\mathrm{OH}(1)$ vibrational frequencies } \\
\cline { 3 - 4 } Mode & AMU & $\mathrm{cm}^{-1}$ & $\mathrm{meV}$ \\
\hline Stretching & 1.08 & 3674 & 456 \\
Rotation I & 1.09 & 919 & 114 \\
Rotation II & 1.15 & 678 & 84 \\
Translation I & 12.13 & 481 & 60 \\
Translation II & 6.90 & 439 & 54 \\
Translation III & 3.85 & 292 & 36 \\
\hline \hline
\end{tabular}

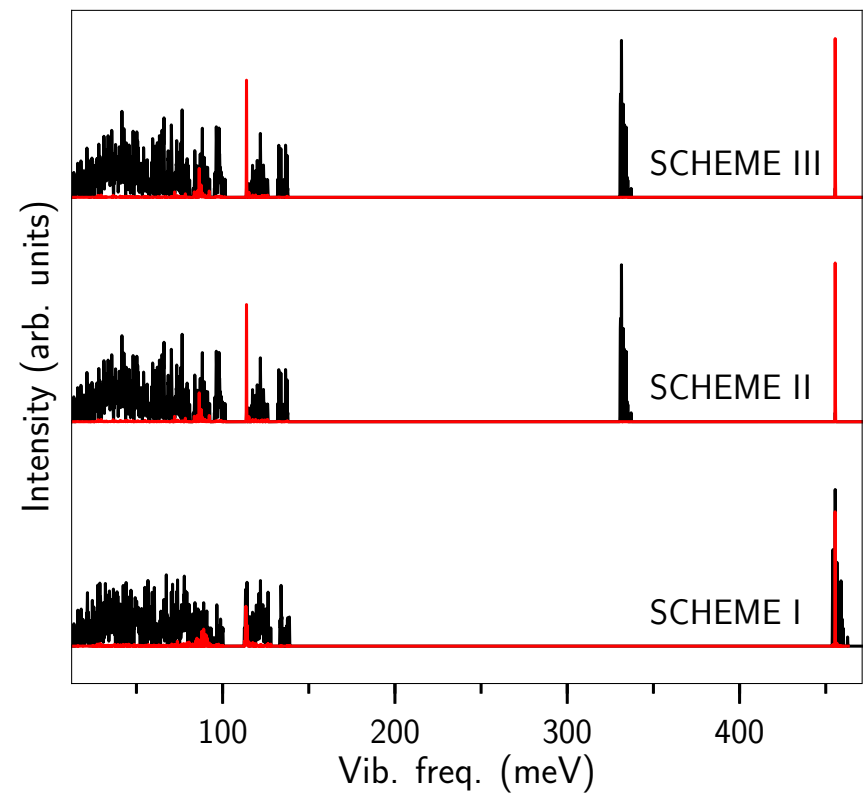

FIG. 11. Visualization of the total phonon density of states (DOS) (black) for schemes I-III compared to the corresponding partial phonon DOS of the OH(1) group (red). We see that the vibrational frequencies for the $\mathrm{OH}(1)$ group remain unaffected by the choice of normal-mode scheme. However, when comparing the total DOS of Scheme I to Schemes II and III, we see shifts of the vibrational frequencies as a result of the mass-weighting on the environment. These changes are immaterial for the analysis, since the vibrational modes of the core-excited species remain unaffected, apart from the dispersion at lower frequencies, as noted in the discussion in connection to Figs. 6(a)-6(f). 
[1] S. Schreck, A. Pietzsch, B. Kennedy, C. Såthe, P. S. Miedema, S. Techert, V. N. Strocov, T. Schmitt, F. Hennies, J.-E. Rubensson, and A. Föhlisch, Sci. Rep. 7, 20054 (2016).

[2] F. Hennies, A. Pietzsch, M. Berglund, A. Föhlisch, T. Schmitt, V. Strocov, H. O. Karlsson, J. Andersson, and J.-E. Rubensson, Phys. Rev. Lett. 104, 193002 (2010).

[3] A. Pietzsch, Y.-P. Sun, F. Hennies, Z. Rinkevicius, H. O. Karlsson, T. Schmitt, V. N. Strocov, J. Andersson, B. Kennedy, J. Schlappa, A. Föhlisch, J.-E. Rubensson, and F. Gel'mukhanov, Phys. Rev. Lett. 106, 153004 (2011).

[4] Y.-P. Sun, A. Pietzsch, F. Hennies, Z. Rinkevicius, H. O. Karlsson, T. Schmitt, V. N. Strocov, J. Andersson, B. Kennedy, J. Schlappa, A. Föhlisch, F. Gel'mukhanov, and J.-E. Rubensson, J. Phys. B 44, 161002 (2011).

[5] Y.-P. Sun, F. Hennies, A. Pietzsch, B. Kennedy, T. Schmitt, V. N. Strocov, J. Andersson, M. Berglund, J.-E. Rubensson, K. Aidas, F. Gel'mukhanov, M. Odelius, and A. Föhlisch, Phys. Rev. B 84, 132202 (2011).

[6] A. Pietzsch, F. Hennies, P. S. Miedema, B. Kennedy, J. Schlappa, T. Schmitt, V. N. Strocov, and A. Föhlisch, Phys. Rev. Lett. 114, 088302 (2015).

[7] J.-E. Rubensson, J. Söderström, C. Binggeli, J. Gråsjö, J. Andersson, C. Såthe, F. Hennies, V. Bisogni, Y. Huang, P. Olalde, T. Schmitt, V. N. Strocov, A. Föhlisch, B. Kennedy, and A. Pietzsch, Phys. Rev. Lett. 114, 133001 (2015).

[8] J.-E. Rubensson, A. Pietzsch, and F. Hennies, J. Electron. Spectrosc. Relat. Phenom. 185, 294 (2012), Special Issue in honor of Prof. T. Darrah Thomas: High-Resolution Spectroscopy of Isolated Species.

[9] J.-E. Rubensson, F. Hennies, and A. Pietzsch, J. Electron. Spectrosc. Relat. Phenom. 188, 79 (2013).

[10] M. Odelius, H. Ogasawara, D. Nordlund, O. Fuchs, L. Weinhardt, F. Maier, E. Umbach, C. Heske, Y. Zubavichus, M. Grunze, J. D. Denlinger, L. G. M. Pettersson, and A. Nilsson, Phys. Rev. Lett. 94, 227401 (2005).

[11] M. Odelius, Phys. Rev. B 79, 144204 (2009).

[12] M. Odelius, J. Phys. Chem. A 113, 8176 (2009).

[13] L. Weinhardt, E. Ertan, M. Iannuzzi, M. Weigand, O. Fuchs, M. Bär, M. Blum, J. D. Denlinger, W. Yang, E. Umbach, M. Odelius, and C. Heske, Phys. Chem. Chem. Phys. 17, 27145 (2015).

[14] M. Blum, M. Odelius, L. Weinhardt, S. Pookpanratana, M. Bär, Y. Zhang, O. Fuchs, W. Yang, E. Umbach, and C. Heske, J. Phys. Chem. B 116, 13757 (2012).

[15] M. Brigatti, E. Galán, and B. Theng, in Handbook of Clay Science, edited by F. Bergaya and G. Lagaly, Developments in Clay Science Vol. 5 (Elsevier, New York, 2013), pp. 21-81.

[16] R. B. Neder, M. Burghammer, T. Grasl, H. Schulz, A. Bram, and S. Fiedler, Clays Clay Miner. 47, 487 (1999).

[17] CPMD, Version 3.15 copyright 2000-2017, IBM Corp. and Max Planck Institute Stuttgart, http://www.cpmd.org (2000-2017).
[18] A. D. Becke, Phys. Rev. A 38, 3098 (1988).

[19] C. Lee, W. Yang, and R. G. Parr, Phys. Rev. B 37, 785 (1988).

[20] N. Troullier and J. L. Martins, Phys. Rev. B 43, 1993 (1991).

[21] C. Hartwigsen, S. Goedecker, and J. Hutter, Phys. Rev. B 58, 3641 (1998).

[22] S. Goedecker, M. Teter, and J. Hutter, Phys. Rev. B 54, 1703 (1996).

[23] $\mathrm{CP} 2 \mathrm{~K}$, Version 2.3 the $\mathrm{CP} 2 \mathrm{~K}$ developers group, https://www.cp2k.org (2013).

[24] M. Iannuzzi and J. Hutter, Phys. Chem. Chem. Phys. 9, 1599 (2007).

[25] J. Hutter, M. Iannuzzi, F. Schiffmann, and J. VandeVondele, Wiley Interdiscip. Rev.: Comput. Mol. Sci. 4, 15 (2014).

[26] M. Krack, Theor. Chem. Acc. 114, 145 (2005).

[27] M. Krack and M. Parrinello, Phys. Chem. Chem. Phys. 2, 2105 (2000).

[28] J. VandeVondele and J. Hutter, J. Chem. Phys. 118, 4365 (2003).

[29] U. Borstnik, J. VandeVondele, V. Weber, and J. Hutter, Parallel Comput. 40, 47 (2014).

[30] J. VandeVondele and J. Hutter, J. Chem. Phys. 127, 114105 (2007).

[31] P. Salek, Comput. Phys. Commun. 150, 85 (2003).

[32] H. A. Kramers and W. Heisenberg, Z. Phys. 31, 681 (1925).

[33] P. Salek, A. Baev, F. Gel'mukhanov, and H. Ågren, Phys. Chem. Chem. Phys. 5, 1 (2003).

[34] M. O. Krause, J. Phys. Chem. Ref. Data 8, 307 (1979).

[35] M. Coreno, M. de Simone, K. Prince, R. Richter, M. Vondráček, L. Avaldi, and R. Camilloni, Chem. Phys. Lett. 306, 269 (1999).

[36] R. Sankari, M. Ehara, H. Nakatsuji, Y. Senba, K. Hosokawa, H. Yoshida, A. D. Fanis, Y. Tamenori, S. Aksela, and K. Ueda, Chem. Phys. Lett. 380, 647 (2003).

[37] G. Ghiringhelli, A. Piazzalunga, C. Dallera, G. Trezzi, L. Braicovich, T. Schmitt, V. N. Strocov, R. Betemps, L. Patthey, X. Wang, and M. Grioni, Rev. Sci. Instrum. 77, 113108 (2006).

[38] V. N. Strocov, T. Schmitt, U. Flechsig, T. Schmidt, A. Imhof, Q. Chen, J. Raabe, R. Betemps, D. Zimoch, J. Krempasky, X. Wang, M. Grioni, A. Piazzalunga, and L. Patthey, J. Synchrotron Radiat. 17, 631 (2010).

[39] A. S.-C. Cheung, K. Yoshino, J. R. Esmond, and W. H Parkinson, J. Mol. Spectrosc. 178, 66 (1996).

[40] A. Pietzsch, J. Nisar, E. Jämstorp, J. Gråsjö, C. Århammar, R. Ahuja, and J.-E. Rubensson, J. Electron. Spectrosc. Relat. Phenom. 202, 11 (2015).

[41] P. Wernet, D. Nordlund, U. Bergmann, M. Cavalleri, M. Odelius, H. Ogasawara, L. A. Näslund, T. K. Hirsch, L. Ojamäe, P. Glatzel, L. G. M. Pettersson, and A. Nilsson, Science 304, 995 (2004).

[42] V. Kimberg and C. Miron, J. Electron. Spectrosc. Relat. Phenom. 195, 301 (2014). 